\title{
A method for a precautionary assessment of aquifer vulnerability to pollution
}

\author{
E. Cameron ${ }^{1}$, L. Garavaglia ${ }^{2}$ \& G. F. Peloso ${ }^{3}$ \\ ${ }^{1}$ GeoStudio, Morbegno (SO), Italy \\ ${ }^{2}$ Department of Environmental Engineering, University of Pavia, Italy \\ ${ }^{3}$ Department of Earth Science, University of Pavia, Italy
}

\begin{abstract}
The assessment of aquifer vulnerability to pollution is an important tool for protecting groundwater resources. Vulnerability may be underestimated due to uncertainties affecting this assessment, with potential adverse consequences for pollution prevention policies; in order to reduce the likelihood of vulnerability underestimation and protect the efficacy of such policies, a precautionary vulnerability value may be used as a reference for planning and design. This paper illustrates an approach for selecting such a precautionary vulnerability value, applied to the vulnerability point count system SINTACS R5 developed by the Italian National Research Council. The method uses possibility theory and fuzzy mathematics in order to: 1) define quantitative models of the uncertainties - including those of a subjective nature - regarding the parameters and partial ratings used to assess vulnerability; 2) to propagate the uncertainty to the final degree of vulnerability; 3 ) to calculate how much each vulnerability degree among the possible ones can be considered precautionary, starting from the definition of the level of precaution related to each degree. Vulnerability maps that correspond to different precaution degrees and can be generated automatically are illustrated, and some possible criteria for selecting an optimal degree are outlined.

Keywords: aquifer vulnerability, uncertainty, precaution, fuzzy mathematics, possibility theory.
\end{abstract}




\section{Introduction}

The assessment of aquifer vulnerability to pollution is an important factor in defining pollution prevention policies aimed at protecting groundwater resources.

This paper considers the assessment method SINTACS R5 (Civita and De Maio [5]) developed by the Italian National Research Council after DRASTIC (Aller et al. [1]). SINTACS is a point count system expressing vulnerability as a degree calculated from the values of seven parameters - from water table depth to topographic surface slope - each indicated by a letter of the acronym. A partial rating is associated to each parameter and then multiplied by a weighting factor related to the hydrogeological setting, the land use and therefore the expected impacts on groundwaters in each part of the territory under consideration. Finally all products are summed to obtain a vulnerability degree ranging from 26 to 260, which can be normalized on a scale from 0 to 100 . In this way each polygonal element in which such territory can be subdivided is assigned to a vulnerability class, from very low to extremely high.

There will normally be some uncertainty as to the parameter values and therefore as to the partial vulnerability rating and the final vulnerability degree, such uncertainty being primarily related to the inherent complexity of most hydrogeological situations, to possible problems with data quality and quantity, to estimation errors and to the partly subjective choice of some ratings.

The term uncertainty, in this paper, denotes a situation in which there is no single possible choice for a parameter value or a partial rating, but a plurality of values and ratings which are to some extent plausible.

Because of uncertainty, therefore, there is also a range of plausible vulnerability degrees, and this raises the question of what degree and class among the possible ones should be assigned to a given area, in order to reduce the likelihood of underestimating vulnerability, unfavourably misclassifying such an area and ultimately decreasing the efficacy of pollution prevention policies.

A possible solution to this problem is the choice of a precautionary vulnerability degree with the approach illustrated in this paper, which makes use of possibility theory and fuzzy mathematics in order to: 1) define quantitative models of the uncertainties - including those of a subjective nature - regarding the parameters and partial ratings used to assess vulnerability; 2) to propagate the uncertainty to the final degree of vulnerability; 3) to calculate how much each vulnerability degree among the possible ones can be considered precautionary, starting from the definition of the level of precaution related to each degree.

This method allows a vulnerability map to be generated automatically in correspondence with each precaution degree. This degree can be appropriately chosen - typically through socio-economical criteria - during the process of formulating a pollution prevention policy, leading to subsequent regulatory decisions. The method, consequently, may also be regarded as a support tool for such a process. 


\section{Brief mathematical outline}

Possibility theory and fuzzy set theory, originally developed at the end of the sixties by the mathematician Lotfi Zadeh, are useful tools for representing non probabilistic uncertainty. The main concepts used in this paper are briefly outlined below; for a full discussion of these subjects the reader is referred to specialized texts (see for example Dubois and Prade [6, 7]).

A fuzzy set $S$, the elements of which are taken from some universal set $U$, is represented by a function $\mu_{S}: U \rightarrow[0,1]$ - called the membership function of $S-$ that associates to an element $x$ of $U$ its membership degree $\mu_{S}(x)$ to $S$, the latter ranging from 0 to 1 and expressing to what extent $x$ is considered to belong to $S$; if $\mu_{S}(x)=0$ then $x$ does not belong to $S$, if $\mu_{S}(x)=1$ then $x$ belongs to $S$ as in the ordinary set theory, while if $0<\mu_{S}(x)<1$ then $x$ belongs to $S$ to the degree given by $\mu_{S}(x)$.

If the fuzzy set $S$ is characterized by a property $P$ then $\mu_{S}(x)$ is also interpreted as the extent to which $x$ satisfies $P$ or is compatible with the concept that $P$ expresses. Let us consider, for instance, the fuzzy set $H$ defined by a membership function $\mu_{H}$ and containing the hydraulic conductivity values - in $\mathrm{m} / \mathrm{s}$ - having the property of being high; then, for example, it may be $\mu_{H}\left(10^{-12}\right)$ meaning that a conductivity of $10^{-12} \mathrm{~m} / \mathrm{s}$ is not considered high at all, $\mu_{H}\left(10^{-2}\right)=1$ meaning that a value of $10^{-2} \mathrm{~m} / \mathrm{s}$ is fully compatible with the concept of high conductivity and $\mu_{H}\left(10^{-6}\right)=0.5$ indicating that $10^{-6} \mathrm{~m} / \mathrm{s}$ is only half compatible with such concept.

The choice of the membership function describing a fuzzy set depends on the concept to be represented and, usually, is not unique and involves some subjectivity; this is not a drawback as fuzzy sets are intended to be formalized models of intrinsically vague and essentially linguistically expressed properties and concepts, such as that of being high with reference to hydraulic conductivity. They are particularly suitable for representing uncertainty regarding nonstatistical or non-measurable properties or objects, but can be usefully employed also for describing imprecisely known quantities; in particular - for the latter purpose - an extensive use is made in this paper of so-called fuzzy numbers, which will shortly be described after some preliminary notions.

The membership function $\mu_{S}$ of a fuzzy set $S$ is said to be normalized if it reaches 1 for at least one element $x$ of the universal set $U$ from which $S$ is defined. The support of $S$ is the ordinary set of all elements of $U$ for which $\mu_{S}(x)>0$, while an $\alpha$-cut of $S$ is the ordinary set of all elements of $U$ for which $\mu_{S}(x) \geq \alpha$.

A fuzzy number is a fuzzy set, defined on the real line $R$, with a normalized membership function $\mu$, a unique modal value - i.e. $\mu(x)=1$ for one and only one $x \in U-$ a bounded support and $\alpha$-cuts, for $\alpha \in(0,1]$, which are all closed 
intervals of $R$. If $R$ contains the values that a variable $v$ may take, then the membership degree $\mu(x)$ can be interpreted as the possibility $\pi(x)$ that $v$ takes the value $x$. The definition and properties of possibility measure - and its dual, called necessity measure - are the subject of possibility theory; here it suffices to say that if $\mu(x)=0$ then $v$ cannot possibly take the value $x$, if $\mu(x)=1$ then it is fully possible - although not necessarily certain - that $v$ takes the value $x$, while if $0<\mu(x)<1$ then there is an intermediate possibility - given by $\mu(x)$ - that $v$ takes the value $x$. A fuzzy number - and in general any fuzzy set - can therefore be thought of as a possibility distribution; an example regarding water table depth is shown in figure 1.



Figure 1: A triangular fuzzy number expressing uncertainty as to the depth $d$ of a water table; $\mu_{D}$ is the membership function.

Fuzzy numbers are non-probabilistic representations of approximately known quantities and subjective evaluations of the latter. The highest membership degree/possibility of 1 is assigned to the best estimate or most plausible value of a variable $-15 \mathrm{~m}$ for depth $d$ in figure 1 - while the lower and upper bounds of the support $-7 \mathrm{~m}$ and $20 \mathrm{~m}$ in figure 1 - indicate, respectively, the lowest and highest possible value that such a variable may take. Fuzzy numbers convey the information that the variable has a value "around" its best estimate, for instance again with reference to figure 1 - that the water table depth $d$ is around $15 \mathrm{~m}$.

A membership function reaching 1 for a number $x$ and being 0 otherwise indicates that a variable $v$ certainly takes the value $x$, since any other is impossible; a membership function reaching 1 for all values that $v$ may take expresses a state of total ignorance as to the value of $v$, because each one is equally and fully possible.

In this paper fuzzy numbers are used throughout for expressing uncertainty as to vulnerability parameters as well as partial ratings and the final degree, so as to 
use a single mathematical tool for treating uncertainty and to exploit fuzzy number suitability for representing subjective uncertainty; in the latter case the membership degree/possibility degree expresses the plausibility assigned to a value or partial rating by the person assessing vulnerability.

Fuzzy arithmetic, starting with the four basic operations, has been developed for fuzzy numbers, with ordinary real numbers being treated as special cases of fuzzy ones. In general many concepts of mathematics have been adapted to allow fuzzy entities to be dealt with.

Fuzzy uncertainty can be propagated via functional relationships by means of the so-called extension principle; if $A$ is a fuzzy set with membership function $\mu_{A}$ and $f$ is a function defined on $A$ and taking its values in a set $B$, then $f$ induces a fuzzy set in $B$, the membership function $\mu_{B}$ of which is defined for every $y \in B$ by:

$$
\mu_{B}(y)= \begin{cases}\sup \left\{\mu_{A}\left(x \mid x \in f^{-1}(y)\right)\right\} & \text { if } f^{-1}(y) \neq \varnothing \\ 0 & \text { if } f^{-1}(y)=\varnothing\end{cases}
$$

where $f^{-1}(y)$ is the set containing all the elements of $A$, if any, to which $y$ is associated by $f$.

The problem of assigning possibility values, transforming probabilities into possibilities and vice versa or treating the two together has also been investigated. It is beyond the scopes of this paper to discuss these issues; reference to them is made, where necessary, in the following paragraphs.

\section{SINTACS}

The parameters considered by SINTACS for assessing vulnerability are: water table depth, yearly aquifer recharge from rainfall, unsaturated zone lithology, soil type, aquifer lithology, hydraulic conductivity and topographic surface slope. These parameters control the ease with which a pollutant can reach an aquifer and the self-depuration effects taking place in the soil, the unsaturated zone and the aquifer itself.

The investigated area is subdivided in discrete elements called cells, in which the hydrogeological situation - with respect to vulnerability assessment - is considered uniform. From the values taken by the parameters within each cell a partial vulnerability rating between 1 and 10 is obtained in two ways: 1) through functions associating each value with a rating for water table depth, recharge from rainfall, hydraulic conductivity and topographic surface slope; 2) through relations associating a value with a range of possible ratings for unsaturated zone lithology, soil type and aquifer lithology. Similar relations are used for estimating the infiltration coefficient from the soil texture or the hydraulic conductivity from the aquifer lithology. The appropriate partial rating or factor value is, in this case, inevitably chosen with a degree of subjectivity. 
The importance of each parameter in the calculation of the final vulnerability degree is determined by a weighting factor ranging from 1 to 5 . The weighting factors depend on the hydrogeological setting, the land use and therefore the expected impacts on groundwaters; they are given for standard situations but may be modified by the user provided their sum is 26 .

If $v_{i}-i=1, \ldots, 7-$ is the partial rating associated to parameter $i$ and $p_{i}$ is the weighting factor of the latter then the final vulnerability degree $V$ - from 26 to 260 - is calculated as:

$$
V=\sum_{i=1}^{7} p_{i} v_{i}
$$

A normalized degree $V_{n}$ - from 0 to 100 - is obtained as:

$$
V_{n}=\left(\frac{V-26}{260-26}\right) \cdot 100
$$

Each cell can be assigned to a vulnerability class as indicated in table 1 . The regulations concerning groundwater protection will normally depend on this class, instead of the specific degree. For example, as part of a study conducted by the Province of Venice [10] the correspondence between the vulnerability classes and the allowable quantity of zootechnical fertilizers - shown in the last column of table 1 - was suggested as a reference point for ensuring a degree of protection of groundwaters from contamination by nitrates coming from agricultural activities.

Table 1: Cell vulnerability class based on the vulnerability degree and correspondence between vulnerability classes and allowable quantities of zootechnical fertilizers.

\begin{tabular}{|c|c|c|c|}
\hline $\begin{array}{c}\text { Vulnerability } \\
\text { degree }\end{array}$ & $\begin{array}{c}\text { Normalized } \\
\text { vulnerability } \\
\text { degree }\end{array}$ & $\begin{array}{c}\text { Vulnerability } \\
\text { class }\end{array}$ & $\begin{array}{c}\text { Zootechnical } \\
\text { fertilizers (kg of } \\
\text { nitrogen/hectare/year) }\end{array}$ \\
\hline $26-80$ & $0-24$ & Very low & 340 \\
\hline $81-105$ & $25-35$ & Low & 340 \\
\hline $106-140$ & $36-49$ & Medium & 250 \\
\hline $141-186$ & $50-69$ & High & 250 \\
\hline $187-210$ & $70-79$ & Very high & 170 \\
\hline $211-260$ & $80-100$ & Extremely high & 0 \\
\hline
\end{tabular}

By assigning a colour or a texture to each vulnerability class and therefore to each cell vulnerability maps are generated. For complete descriptions and application examples of SINTACS R5 the reader is referred, first of all, to Civita and De Maio [5]. 


\section{Precautionary vulnerability assessment}

The precautionary assessment of vulnerability illustrated in this paper starts with the description - by means of triangular fuzzy numbers - of the uncertainty: 1) as to the depth of the water table, as in figure 1, the hydraulic conductivity and the topographic surface slope; 2) as to the infiltration coefficient used for estimating an aquifer yearly recharge from rainfall; 3 ) as to the partial ratings related to soil type, unsaturated zone lithology and aquifer lithology.

For case 1) the uncertainty is directly propagated to the partial ratings through the extension principle, since parameter values and such ratings are related by functions. For case 2) the fuzzy number relative to the infiltration coefficient is multiplied by the real number representing the yearly average rainfall or the yearly net rainfall after evapotranspiration, depending on whether the soil thickness is equal to or more than one meter, or less than one meter; the resulting fuzzy number describes the uncertainty as to the amount of recharge, which is then propagated to the relative partial rating again by making use of the extension principle. For case 3), finally, the subjective uncertainty as to the partial rating to be assigned to a soil type or a lithology is represented by a fuzzy number, the membership degrees of which express to what extent each rating is considered plausible given such soil type or lithology.

By applying this procedure the seven partial ratings $v_{i}-i=1, \ldots, 7$ - become fuzzy numbers expressing the possibility assigned to each rating, given the uncertainty as to the input data. Such fuzzy numbers are multiplied by the respective weighting factors $p_{i}$ to obtain the final vulnerability degree - again a fuzzy number - according to eqn. (2).

The uncertainty as to the value of a measurable parameter will be, in many cases, initially expressed in probabilistical/statistical terms. For instance by using ordinary kriging the depth of the water table may be evaluated as $d \pm \Delta d$, where $d$ is the estimate and $\Delta d$ is such that the probability of the actual depth being outside the interval $[d-\Delta d, d+\Delta d]$ is lower than a value $p^{\prime}$. In this case the simplest - although to some extent arbitrary - choice for defining the correspondent fuzzy number may be to take $\Delta d$ so that $p^{\prime}$ is very low (ideally negligible), to consider $d$ as the most plausible value, having a membership degree of 1 , and to set the open interval $(d-\Delta d, d+\Delta d)$ as the fuzzy number support. Several papers deal with the problem of relating uncertainty expressed by means of probability and possibility theory or obtaining possibility distributions, and the reader is referred to them (for example Bárdossy and Fodor [2], Masson and Denœux [9], Raufaste et al. [11]).

What is more, some of the parameters- such as aquifer lithology and hydraulic conductivity - and the relative partial ratings are related to each other; methods have been developed for extending fuzzy arithmetic to the so-called "interactive variables" such as those just mentioned (see for instance Lesage [8]).

Finally the description of uncertainty by fuzzy numbers has been limited - at present - to the cases considered above, but in principle such a description may 
also regard the evaluation of rainfall or evapotranspiration as well as the weighting factors used for obtaining the final vulnerability degree.

The fuzzy number representing the uncertainty as to the final vulnerability degree will be similar to that in figure 2, although it will not be exactly triangular because of the shape of the function correlating water table depth, recharge and hydraulic conductivity to their partial ratings.

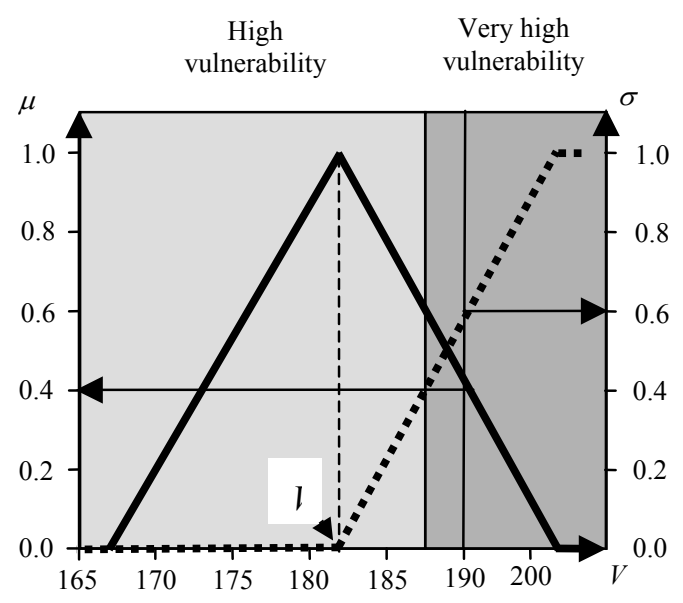

Figure 2: A fuzzy number (bold line) expressing uncertainty as to the vulnerability degree $V$. The membership degree $\mu$ gives the possibility attributed to each vulnerability degree. The right scale indicates the precaution degree $\sigma$ (dotted line) and the arrows show an example of its calculation for a vulnerability degree equal to 190 (see text). The ranges of vulnerability degrees belonging to the high and very high vulnerability classes are shown with a lighter and darker background respectively.

Figure 2 shows that although the most plausible estimate $\hat{V}$ of the vulnerability degree is 182 , higher degrees are significantly plausible, and those just above the transition to the very high vulnerability class are considerably possible, as indicated by a possibility that is still around 0.6.

The more precautionary a vulnerability degree $V^{*}$, chosen in the range $26-$ 260 , is considered to be, the less is the possibility $\pi\left(V>V^{*}\right)$ of the vulnerability degree $V$ being higher than $V^{*}$, given by $\pi\left(V>V^{*}\right)=\sup \left\{\mu(V) \mid V>V^{*}\right\}$. For fuzzy numbers similar to that in figure 2 such possibility is:

$$
\pi\left(V>V^{*}\right)= \begin{cases}1 & \text { for } V \leq \hat{V} \\ \mu(V) & \text { for } V>\hat{V}\end{cases}
$$


taking $\pi(V>260)=0$ since the vulnerability degree cannot be higher than 260 .

The precaution degree $\sigma\left(V^{*}\right)$ related to the choice of $V^{*}$ is defined as $\sigma\left(V^{*}\right)=1-\pi\left(V>V^{*}\right)$ so that in this case it is:

$$
\sigma\left(V^{*}\right)= \begin{cases}0 & \text { for } V \leq \hat{V} \\ 1-\mu(V) & \text { for } V>\hat{V}\end{cases}
$$

taking $\sigma(260)=1$ since the vulnerability degree cannot be higher than 260 .

From figure 2 it can be deduced that, according to eqn (4) and eqn (5), $\sigma(160)=1-\pi(V>160)=1-1=0$, meaning that choosing a vulnerability degree equal to 160 is not precautionary at all, in accordance with the fact that the latter figure is lower than the best estimate of 182; at the same time, for example, $\sigma(190)=1-\pi(V>190)=1-0.4=0.6$ and, therefore, attributing to the cell a vulnerability degree of 190 is fairly precautionary, because the possibility of such a degree being higher than 190 - equal to 0.4 - is rather low. Finally $\sigma(202)=1-\pi(V>202)=1-0=1$ : considering the vulnerability degree of the cell equal to 202 is totally precautionary, because such a degree cannot possibly be higher than the latter figure. The precaution degree corresponding to $\hat{V}=182$ is 0: attributing to a cell the best estimate of the vulnerability degree is surely justified, but strictly speaking not precautionary if such a degree is possibly higher than the best estimate itself.

By arranging the vulnerability classes in ascending order, from very low to extremely high, the concept of precaution degree can be extended to such classes. The possibility $\pi\left(C>C^{*}\right)$ that the vulnerability degree belongs to a class $C$ higher than the one $-C^{*}$ - attributed to a particular cell, is given by $\pi\left(C>C^{*}\right)=\pi\left(V>V_{C^{*}}\right)$, where $V_{C^{*}}$ is the degree representing the upper bound of $C^{*}$. The precaution degree related to the choice of the class $C^{*}$ for the cell can then be expressed as $\sigma\left(C^{*}\right)=1-\pi\left(C>C^{*}\right)=1-\pi\left(V>V_{C^{*}}\right)$.

From figure 2 it can then be deduced that the precaution degree related to classifying the cell in the high vulnerability class is 0.4 (the reader should follow the lower arrow from the dotted line) and hence rather low, because it is significantly possible that vulnerability degrees belong to the very high vulnerability class; choosing the latter for the cell is totally precautionary, as vulnerability degrees greater than the upper bound of the class (210, see table 2) are not considered possible.

Because the relationship between the vulnerability degree and the precaution degree is one-to-one over most of the fuzzy number domain, then the latter degree may be chosen for each cell as an input for calculating the related vulnerability degree and attributing the cell to the corresponding class. 
Figure 3 shows two maps relative to the territory of Vigevano (Province of Pavia, Italy, extension $81 \mathrm{~km}^{2}$ ) calculated for two precaution degrees which are uniform over the entire area.


Figure 3: Vulnerability maps for a precaution degree equal to 0 (left) and 0.5 (right). Legend: \igh vulnerability; very high vulnerability; extremely high vulnerability.

Already for a precaution degree equal to 0.5 the cells attributed to the high vulnerability class are almost halved with a reduction in area of about $12.5 \mathrm{~km}^{2}$, while a relevant quantity of those belonging to the extremely high class appear over an area around $15 \mathrm{~km}^{2}$. With reference to table 2 and the agricultural areas of the territory under consideration this would imply a reduction of fertilization with zootechnical fertilizers from 250 to $170 \mathrm{~kg}$ of nitrogen/hectare/year over a significant extension, while in other sectors fertilization as such should be regarded, theoretically, as incompatible with groundwater protection.

The choice of the precaution degree on which to base a pollution prevention policy typically pertains to the final - and usually political - decision making level, while specifying the uncertainties affecting vulnerability assessment is chiefly a technical problem.

A very simple criterion, not requiring cost-benefit analysis, is to choose that vulnerability degree/precaution degree pair representing the optimal trade-off between precaution and plausibility. Such a pair, in figure 2, corresponds to the intersection between the bold line of the fuzzy set expressing the possibility distribution of the vulnerability degrees and the dotted line giving the related precaution degrees; the vulnerability degree so obtained is about 188 and the relative precaution degree is 0.5 . Higher vulnerability degrees are more precautionary but less plausible, as $\sigma$ increases but $\mu$ decreases, and vice versa for lower vulnerability degrees. A vulnerability degree of 188 would cause an attribution of the cell to the extremely high vulnerability class, which corresponds to total precaution.

In principle, the choice of the optimal precaution degree should be based on an analysis of the expected costs - or lost benefits - related to land use or design constraints due to each vulnerability degree and/or class corresponding to the 
precaution degree, and the expected benefits of the consequent groundwater resources protection.

An approach similar to the one described in this paper may be applied to the precautionary assessment of other risk factors, even where the uncertain quantity is the likelihood - say the probability - that an event will happen (Cameron and Peloso [4]); the extension of the method to the complete precautionary assessment of risk is currently under development.

\section{References}

[1] Aller, L., Bennet, T., Hackett, G., Lehr, J. H. \& Petty, R. J., DRASTIC. A standardized system for evaluating groundwater pollution potential using hydrogeological settings, North Wales Water Authority/EPA Serv., EPA 600/2 - 85/018: Washington, 1987.

[2] Bárdossy, G. \& Fodor, J., Evaluation of uncertainties and risks in geology, Springer Verlag: Heidelberg, Germany, 2004.

[3] Cameron, E. \& Peloso, G. F., An application of fuzzy logic to the assessment of aquifer's pollution potential. Environmental Geology, 40, pp. 1305-1315, Springer Verlag: Heidelberg, Germany, 2000.

[4] Cameron, E. \& Peloso, G. F., Risk management and the precautionary principle: a fuzzy logic model. Risk Analysis, 25/4, pp. 901-912, Blackwell: Malden, MA, USA, 2005.

[5] Civita, M. \& De Maio, M., SINTACS: un metodo parametrico per la valutazione e la cartografia della vulnerabilità degli acquiferi all'inquinamento, Pitagora Editrice: Bologna, Italy, 2001.

[6] Dubois, E. \& Prade, H. M., Possibility theory, Plenum Press, New York, USA, 1988.

[7] Dubois, E. \& Prade, H. M., (eds). The handbook of fuzzy sets volume 7: fundamentals of fuzzy sets, Kluwer Academic Publisher: Dordrecht, The Netherlands, 2000.

[8] Lesage, C., Discounted cash-flow analysis: an interactive fuzzy arithmetic approach. European Journal of Economic and Social Systems, 15/2, pp. 46 - 68, EDP Sciences: Les Ulis, France, 2001.

[9] Masson, MH. \& Denœux, T., Inferring a possibility distribution from empirical data. Fuzzy Sets and Systems, 193/3, pp. 319-340, Elsevier: North-Holland, 2006.

[10] Provincia di Venezia, Cartografia relativa all'attitudine dei suoli allo spargimento dei liquami zootecnici - Carta della vulnerabilità degli acquiferi all'inquinamento, Provincia di Venezia, 2000.

[11] Raufaste, E., Da Silva Neves, R., Mariné, C., Testing the descriptive validity of possibility theory in human judgements of uncertainty. Artificial Intelligence, 148, pp. 197-218, Elsevier: Essex, UK, 2003. 